\title{
Handlungsfähigkeit der EZB nach Draghi
}

Seit 2014 veranstaltet die Europäische Zentralbank (EZB) Mitte Juni ihre jährliche Forschungskonferenz - das ECB Forum on Central Banking - im portugiesischen Sintra. Diesmal, wenige Monate vor dem Ende der Amtszeit Mario Draghis, ging es um eine Bestandsaufnahme der ersten 20 Jahre der Europäischen Währungsunion, insbesondere aber der turbulenten letzten acht Jahre seit seinem Amtsantritt. Viele Beiträge priesen den Pragmatismus und die intellektuelle Kreativität der EZB dabei, wie sie die unkonventionelle Geldpolitik seit Ausbruch der Finanzkrise umgesetzt hatte. Hauptthema war aber, dass die EZB auch künftig bei der Gestaltung ihrer geldpolitischen Maßnahmen mit zentralen Schwächen im Design der Währungsunion zu kämpfen haben wird.

Ein Schwachpunkt ist zum Ersten die bislang noch ausstehende Vollendung der Bankenunion und der Integration der Kapitalmärkte im Euroraum. Hier sind im Laufe der letzten Jahre schon gute Fortschritte erzielt worden. Die Stärkung des einheitlichen Aufsichtsmechanismus der Großbanken im Euroraum sowie die Umsetzung eines einheitlichen Abwicklungsmechanismus durch eine zentrale Behörde, die auf einen gemeinschaftlichen Fonds zurückgreifen kann, sind wichtige Voraussetzungen dafür, Finanzmarktschocks im Euroraum in Zukunft wesentlich wirksamer abzufedern als 2008 - insbesondere dann, wenn sie durch eine gemeinsame Einlagensicherung sowie durch rigorose Anwendung makro-prudenzieller Regulierungsmechanismen auf nationaler Ebene (wie antizyklische Eigenkapitalpuffer) komplementiert werden. Dagegen wird die Effektivität der Geldpolitik weiterhin durch zwei Design-Schwächen begrenzt. Zum einen fehlen ausreichende Mechanismen, um die relativen Preise und Löhne im Euroraum in Phasen niedriger Inflationsraten anzupassen. Die Entwicklung im Lauf der letzten zehn Jahre hat eindrucksvoll bestätigt, welch extrem hohe realwirtschaftliche Kosten die mühsame Anpassung über Deflation mit sich bringt.

Zum Zweiten wird die Geldpolitik im Euroraum durch Unzulänglichkeiten im fiskalpolitischen Rahmenwerk der EU stark eingeengt. Eine wirksame Stabilisierung mit Hilfe der Fiskalpolitik erfordert es, die fiskalpolitischen Maßnahmen der einzelnen Staaten besser zu koordinieren. Solange es an einer solchen Koordination mangelt (und diese Stabilisierung zudem auch nicht alternativ über ein europäisches Budget erfolgen kann), muss die Geldpolitik die alleinige Verantwortung übernehmen. In einer Welt niedriger und teilweise negativer Zinsen ist traditionelle Geldpolitik mit dieser Aufgabe jedoch eindeutig überfordert; die EZB sieht sich ständig zum Experimentieren gezwungen, um diesen Mangel mit immer neuen unkonventionellen Maßnahmen auszugleichen. In der Amtszeit Mario Draghis hat die EZB dabei große Kreativität an den Tag gelegt. Sie hat nicht nur viele erfolgreich von der Fed getestete unkonventionelle Instrumente übernommen (wie die Politik der "Forward Guidance“ sowie der quantitativen Lockerung mit einem massiven Ankauf von Staatsanleihen am Sekundärmarkt), sondern auch selbst innovative Programme in Gang gesetzt, wie das OMT-Programm (Outright Monetary Transactions), die Einführung negativer Zinsen auf Bankeinlagen sowie gezielter längerfristiger Refinanzierungsgeschäfte (LTRO) zur Stimulierung der Kreditvergabe der Banken.

Auch wenn es schwierig ist, die Auswirkung einzelner Programme zu messen (viele Maßnahmen wurden ja parallel eingeführt), zeigen ökonometrische Studien, dass sie zusammengenommen zu einer signifikanten Stabilisierung der Finanzmarktvariablen führten. Trotz aller Stimulierungsmaßnahmen scheint aber der Transmissionsmechanismus auf reale Größen im Vergleich zu den Zeiten vor der Krise eher langsam zu wirken. An der Zinsuntergrenze ist der Einfluss der Geldpolitik auf die reale Kredit-

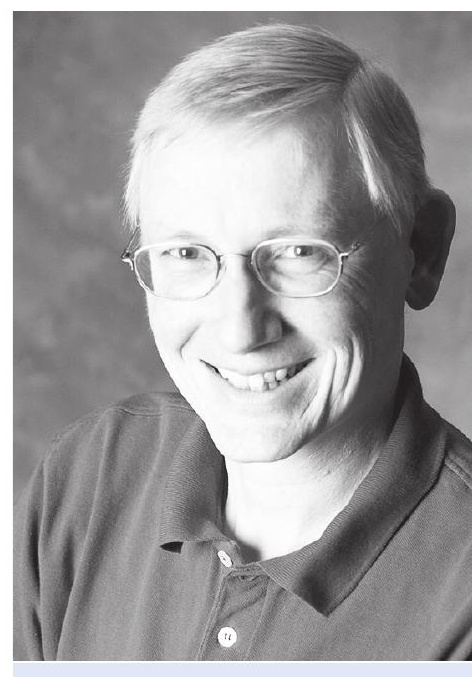

Gerhard Illing ist Professor und leitet das Seminar für Makroökonomie am Institut für Volkswirtschaftslehre der LudwigMaximilians-Universität München. 
vergabe offensichtlich weniger stark als in normalen Zeiten. Hinzu kommt, dass verschärfte Regulierungsmaßnahmen zur Minderung der Risiken im Bankensektor die Kreditvergabe dämpfen und damit kurzfristig gegenläufig zu den unkonventionellen Maßnahmen wirken. Die EZB sah sich mit ihren unkonventionellen Programmen vor allem in der deutschen Öffentlichkeit starker Kritik ausgesetzt. Viele warfen ihr vor, die Niedrigzinspolitik „enteigne“ den deutschen Sparer. Sie übersahen dabei, dass das Zinsniveau weltweit in ungeahnte Tiefen gefallen ist. Niedrige Zinsen sind keineswegs ein Sonderweg der EZB, sondern die „neue Normalität“ in den Industriestaaten.

Im Laufe der letzten Jahre sah es so aus, als würde diese Politik endlich greifen. Die Wirtschaftsentwicklung schien auf gutem Weg zu sein. Im Euroraum zogen die Wachstumsraten an, ohne die Preisstabilität zu gefährden. Der Wirtschaftsboom in den USA verleitete die Fed endlich dazu, aus dem Programm quantitativer Lockerung auszusteigen und die Zinsen langsam anzuheben. Doch in jüngster Zeit hat sich das Bild wieder verdüstert. Die Inversion der Zinsstrukturkurve in den USA deutet auf eine drohende Rezessionsgefahr. Viele Zentralbanken sehen sich derzeit veranlasst, eine Umkehr zu weiter sinkenden Zinsen anzudeuten. Wie groß der Handlungsspielraum der Geldpolitik überhaupt noch ist, bleibt abzuwarten. Schon seit längerem beschäftigt sich die Forschung der Fed intensiv mit der Frage, wie ihre geldpolitische Strategie angepasst werden sollte, um besser gerüstet zu sein, wenn die Zinsen wieder an die Untergrenze stoßen. Bei der Fed findet der Vorschlag immer mehr Anhänger, in einem solchen Fall für längere Zeit ein Überschießen der Inflation über $2 \%$ hinaus explizit anzustreben.

Auch im Euroraum verdüstern sich derzeit die Konjunkturaussichten im Lichte zunehmender Handelskonflikte und verstärkten Protektionismus. Deutschland scheint dabei besonders stark von einer einbrechenden Exportnachfrage betroffen zu sein. Angesichts dieser Eintrübungen betonte Mario Draghi auf der Sintra-Konferenz am 18. Juni 2019, die EZB sei voll handlungsfähig; sie werde alle Flexibilität nutzen, um ihren Auftrag zu erfüllen. Dabei seien sowohl Senkungen des Leitzinses als auch eine Wiederaufnahme des Anleihekaufprogramms denkbar. Manche Kritiker warfen Draghi vor, mit diesen Aussagen enge er den Handlungsspielraum seines Nachfolgers unnötig ein. Doch im Grunde hat Draghi nur etwas Selbstverständliches ausgesprochen: Jeder Nachfolger muss Interesse daran haben, im Fall des Falls alle verfügbare Flexibilität voll ausnutzen zu können. Die entscheidende Frage ist aber, wie handlungsfähig die EZB wirklich sein wird, sollte sich die befürchtete Konjunktureintrübung verfestigen. Schließlich könnten weitere Zinssenkungen in den negativen Bereich durchaus kontraproduktiv wirken, wenn sie den Bankensektor und damit die Finanzmarktstabilität gefährden. Solche Zinsschritte sollten zukünftig daher nur mehr marginale Bankeneinlagen betreffen; der Großteil der bestehenden Einlagen dagegen sollte von negativen Zinsen ausgenommen werden. Andere Zentralbanken (etwa in der Schweiz oder in Japan) verfahren schon seit dem Start ihrer Negativzinspolitik in der Weise. Ein solcher Strategiewechsel könnte den Geschäftsbanken in der Europäischen Währungsunion Freiraum verschaffen, ohne weitere Zinssenkungen auszuschließen. Es ist zu hoffen, dass die neue EZB-Präsidentin offene Ohren für solche Vorschläge hat.

Man sollte sich aber keinen Illusionen hingeben: Selbst höchste intellektuelle Kreativität kann nichts daran ändern, dass der Handlungsspielraum der EZB bei einem neuen Konjunktureinbruch begrenzt sein wird. Ein rascher gezielter Einsatz aktiver Stimulierungsmaßnahmen durch die Fiskalpolitik wird unverzichtbar sein, um die Geldpolitik zu entlasten. Weil nicht nur in Deutschland ohnehin ein massiver Bedarf

Gerhard Illing

Ludwig-Maximilians-Universität

München

Illing@econ.Imu.de für den Ausbau staatlicher Investitionsaktivität besteht, wäre es dringend angeraten, entsprechende Planungen rechtzeitig vorzubereiten. Solche Maßnahmen auf europäischer Ebene zu koordinieren, wird nicht die Aufgabe der EZB sein, sondern der neuen EU-Kommission. 